\title{
Padrão de ponta-onda Contínua durante o sono na epilepsia parcial idiopática: relato de um caso sem alterações neuropsiquiátricas
}

\author{
Idiopathic focal epilepsy and continuous spikes and waves during slow sleep: a case \\ report without neuropsychological impairment
}

\author{
Ângela Rodrigues Gifoni ${ }^{1}$ \\ Marielza Fernandez Veiga ${ }^{2}$ \\ Maria Betânia Pereira Toralles ${ }^{3}$
}

\begin{abstract}
${ }^{1}$ Médica. Mestranda do Programa de Pós-Graduação em Processos Interativos dos Órgãos e Sistemas, ICS - UFBA
${ }^{2}$ Médica Neurofisiologista Clínica. Hospital Universitário Professor Edgard Santos, UFBA

${ }^{3}$ Professora Associado. Departamento de Pediatria, FAMEB - UFBA. Programa de Pós-Graduação Processos Interativos dos Órgãos e Sistemas, ICS - UFBA
\end{abstract}

\begin{abstract}
Resumo
Introdução: A epilepsia benigna da infância com descargas centro-temporais, também conhecida como epilepsia rolândica, é uma epilepsia parcial idiopática que acomete crianças e apresenta evolução favorável, com normalização do padrão eletroencefalográfico durante a adolescência. Alguns casos, entretanto podem apresentar uma evolução atípica, com o surgimento do padrão de ponta-onda contínua durante o sono no EEG e distúrbios neurológicos graves. Objetivo: Descrever os aspectos clínicos, eletroencefalográficos e a resposta ao tratamento de uma paciente com Epilepsia Rolândica que evoluiu de forma atípica, apresentando o padrão eletroencefalográfico de ponta-onda contínua durante o sono, sem alterações neuropsiquiátricas. Metodologia: Relato de caso clínico a partir da obtenção dos dados clínicos e resultados de exames registrados em prontuário médico. Resultados: A paciente inicialmente apresentava um quadro de epilepsia rolândica com manifestações atípicas, após tratamento com Oxcarbazepina evoluiu com padrão ponta-onda contínua no sono no eletroencefalograma e piora na frequência de crises epilépticas. Apresentou melhora transitória com a troca da medicação para Lamotrigina, mas o controle definitivo das crises só ocorreu com o Sulthiame. Em nenhum momento teve alteração cognitiva ou comportamental. Conclusão: A epilepsia benigna da infância com descargas centro-temporais pode apresentar uma evolução com quadro clínico mais grave e a medicação deve ser reavaliada nesses casos. $O$ tratamento deve objetivar o controle das crises e a preservação das funções cognitivas.
\end{abstract}

Palavras chave: Epilepsia rolândica, ponta-onda contínua no sono, eletroencefalograma, alterações neuropsiquiátricas.

\begin{abstract}
Background : Benign childhood epilepsy with centrotemporal spikes or benign rolandic epilepsy is an focal idiopathic epilepsy syndrome that affects children, with a good prognosis and normalization of the EEG during adolescence. However, some cases may present an atypical evolution with the emergence of continuous spike and wave during slow sleep and serious neurological disorders. Objective: Describe the clinical, electroencephalographic aspects and response to treatment of a patient with atypical evolution of rolandic epilepsy presenting continuous spike and wave during slow sleep, without neuropsychiatric disorders. Methodology: Retrospective description of a case of idiopathic partial epilepsy using data from medical records. Results: The patient initially had clinical and electroencephalographic features of atypical rolandic epilepsy. The administration of Oxcarbazepine resulted in increased seizure frequency and worsening of the EEG with continuous spike and wave during slow sleep. Changing the medication Lamotrigine promoted a transient improvement but the final seizure control only occurred after Sulthiame. At no time had cognitive or behavioral change. Conclusion: The benign childhood epilepsy with centrotemporal spikes can occur with more severe clinical pictures and medication should be reevaluated in these cases. Treatment should aim seizure control and preservation of cognitive function.

Keywords: Rolandic epilepsy, Continuous spike and wave during sleep, electroencephalogram, neuropsychiatric disorders.
\end{abstract}

\section{INTRODUÇÃO}

A epilepsia focal da infância com descargas centro-temporais, também conhecida como epilepsia rolândica, faz parte do grupo das epilepsias benignas da infância por ter uma evolução auto-limitada e de bom prognóstico. É caracterizada por crises pouco frequentes, predominan-

Correspondência / Correspondence: Ângela Rodrigues Gifoni. Programa de Pós-graduação Processos Interativos dos órgãos e sistemas. Av. Reitor Miguel Calmon, S/N, Vale do Canela. CEP: 40110-100. Salvador - BA Fone: (71) 3283-8959 E-mail: angelagifoni@yahoo.com.br temente no sono. Geralmente são crises parciais motoras, orofaciais e/ou sensitivas, podendo apresentar generalização secundária. O eletroencefalograma mostra atividade de base normal com espículas centro-temporais, ativadas pelo sono com remissão na adolescência. $(1,2)$. Embora o grupo das epilepsias benignas da infância não apresente comprometimento cognitivo, hoje se sabe que a epilepsia com descargas centro-temporais possui um espectro clínico amplo e alguns pacientes apresentam alterações cognitivas e comportamentais $(3,4)$. 
As formas atípicas e não tão benignas vêm sendo cada vez mais relatadas em pacientes com epilepsia com descarga centro-temporal. (5,6,7). Em alguns casos, o agravamento clínico e eletrográfico ocorre pela introdução de determinadas drogas antiepilépticas, principalmente drogas que agem no canal de sódio. $(8,9)$. Esta evolução atípica ao uso destas drogas pode ser justificada por fatores genéticos, alguns já relatados, responsáveis pela variabilidade fenotípica deste tipo de epilepsia. (10).

A evolução atípica da epilepsia com descargas centro-temporais é caracterizada por um aumento da frequência das crises, ou um aumento da frequência das descargas epileptiformes e lentificação da atividade de base, ou por um comprometimento cognitivo, principalmente na área da linguagem. No extremo mais grave do espectro clínico, as descargas epileptiformes podem ser tão frequentes que ocupam a maior parte do traçado, caracterizando o padrão de ponta-onda contínua durante o sono (POCS).

Embora o termo POCS não tenha uma uniformidade no seu conceito e terminologia, é hoje reconhecido como uma expressão eletroencefalográfica comum a pelo menos três síndromes epilépticas, incluindo a epilepsia atípica focal "benigna" (a forma atípica da epilepsia rolândica), a síndrome de Landau-Kleffner e a encefalopatia epiléptica com ponta-onda contínua do sono. A ideia atual é que estas síndromes façam parte de um espectro fenotípico em que no extremo mais benigno esteja a epilepsia rolândica, auto-limitada, e no extremo mais grave a epilepsia com POCS, formando um continuum clínico (11).

Na maioria das vezes existe uma correlação entre o aumento da frequência das descargas epileptiformes e a piora na frequência de crises e/ou a deterioração cognitiva.

Relatamos, a seguir, o caso de uma criança com características iniciais de uma epilepsia rolândica que evoluiu com piora das crises epilépticas e padrão POCS, agravados pelas drogas antiepilépticas, sem comprometimento cognitivo.

\section{RELATO DE CASO}

Paciente do sexo feminino, com início da epilepsia aos 10 anos e 8 meses (novembro de 2009), com crise do tipo tônico-clônica generalizada no sono. O eletroencefalograma inicial já mostrou a presença de paroxismos epileptiformes muito frequentes, de projeção difusa em ambos os hemisférios cerebrais, predominando nas regiões parieto-temporais, embora apresentasse características de uma epilepsia rolândica benigna caracterizada por atividade de base normal e formação de dipolo tangencial com campo positivo nas regiões anteriores, ativados pelo sono (Figura 1). Não apresentava anormalidades ao exame neurológico, nem dados relevantes nos antecedentes pessoais. História familiar para epilepsia era negativa. A ressonância magnética de encéfalo foi normal. Iniciado o tratamento após a primeira crise com doses baixas de Oxcarbazepina, com ajuste por recidiva das crises motoras focais envolvendo face (desvio da comissura labial e disartria) e clonias do membro superior esquerdo.

Figura 1. Novembro de 2009, sono. Paroxismos de ondas agudas de projeção difusa em ambos os hemisférios cerebrais, predominando nas regiões parieto-temporais, com bissincronia.

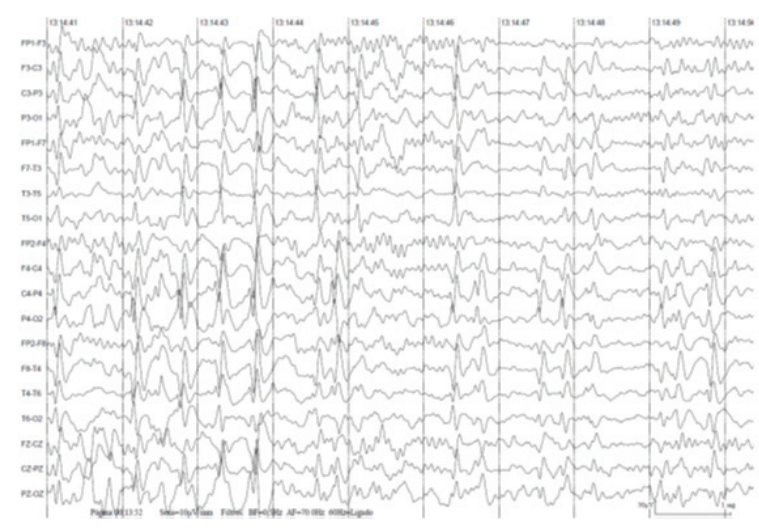

Após dois meses do início do tratamento com Oxcarbazepina (janeiro de 2010), ainda com doses baixas, as crises se tornaram mais frequentes e mais intensas, inclusive com paralisia de Todd, manifestação não observada em epilepsias idiopáticas. As doses da Oxcarbazepina continuaram a ser ajustadas (750-900mg/dia), por recidivas de crises noturnas, quase diárias e com fraqueza motora pós-critica (despertava com desvio da comissura labial). Em março de 2010, passou a apresentar um novo padrão de crise caracterizada por mioclonias negativas diárias, envolvendo o membro superior esquerdo.

Em junho de 2010 ainda apresentava desvio da comissura labial, quase diariamente, sugerindo crises subclínicas durante o sono e piora do traçado eletroencefalográfico com paroxismos epileptiformes de ponta-onda centro-parieto-temporais, com propagação difusa, bilaterais e síncronos, em surtos prolongados, ocupando mais de $50 \%$ do traçado durante o sono (padrão POCS), além de lentificação da atividade de base no hemisfério direito (Figura 2). Nesta época, a Oxcarbazepina foi substituída gradualmente pela Lamotrigina e o EEG realizado (dezembro/10), já em monoterapia com a Lamotrigina, mostrava uma redução acentuada na frequência das descargas epileptiformes com desaparecimento do padrão POCS.

Em junho de 2011 a paciente apresentou recidiva de crises, com as mesmas características e o EEG dessa época confirmava a reativação das descargas epileptiformes difusas e bilaterais. A Lamotrigina foi substituída pelo Sulthiame (Ospolot ${ }^{\circledR}$ ), droga não comercializada no Brasil, e cujo mecanismo de ação difere das duas primeiras drogas.

Após a substituição pelo Sulthiame, a paciente não apresentou mais crises, embora nos traçados de EEG ainda apresentasse o padrão POCS com variação da frequência das descargas epileptiformes (índice espícula-onda), mais 
lateralizadas no hemisfério cerebral direito. Apesar da não melhora inicial do $E E G$, inclusive mantendo o padrão POCS no exame de setembro/11(Figura 3), não houve recidiva de crises e a paciente não apresentava alterações comportamentais nem cognitivas, atestada pelo exame neuropsicológico em dezembro/11.

Figura 2. Junho de 2010, sono. Ponta-onda contínua no sono. Descargas bilaterais e síncronas ocupando em torno de $50 \%$ do traçado durante o sono.

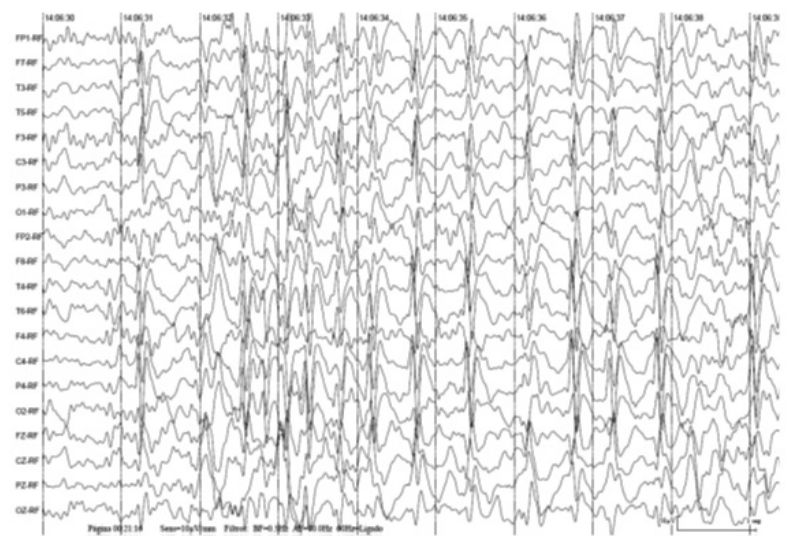

Figura 3. Setembro de 2011, sono. Padrão de ponta-onda contínua no sono com bissincronia e predomínio à direita.

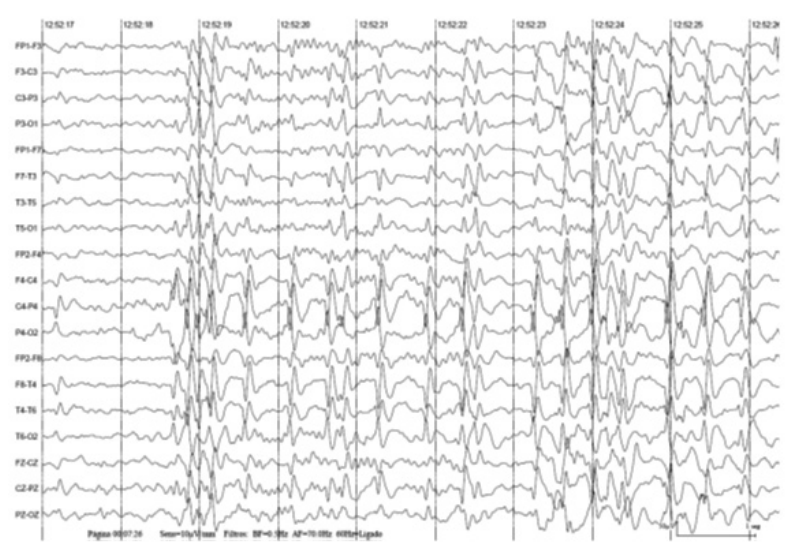

Figura 4. Março de 2013, sono. Mostra o desaparecimento do padrão ponta-onda contínua no sono.

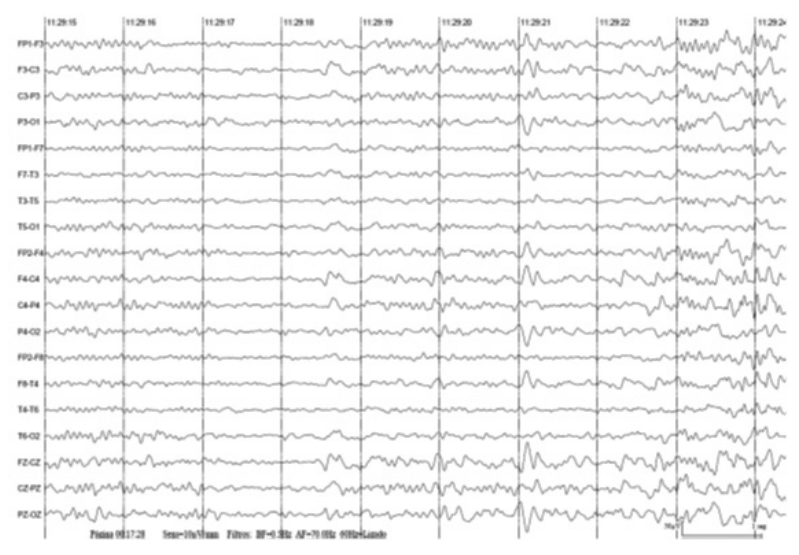

Durante todo o ano de 2013 a paciente não teve qualquer manifestação clínica e o EEG apresentou meIhora acentuada com desaparecimento do padrão POCS e com raras descargas epileptiformes restritas à região centro-parietal direita. (Figura 4).

\section{DISCUSSÃO}

O caso relatado apresenta algumas características clínicas e eletroencefalográficas que configuram manifestações atípicas da epilepsia benigna da infância com descargas centro-temporais, como a presença de paroxismos difusos nos dois hemisférios cerebrais com bissincronia secundária evoluindo para o padrão POCS, a lentificação assimétrica da atividade de base, a recidiva de crises muito frequentes e prolongadas com paralisia de Todd e o surgimento de novo tipo de crise (mioclonias negativas). Apesar desse grupo de pacientes com manifestações atípicas apresentar uma maior percentagem de distúrbios comportamentais e de aprendizagem quando comparado aos pacientes com manifestações típicas (12), a nossa paciente não apresentou alterações neuropsiquiátricas.

Com relação à evolução eletroencefalográfica, o surgimento do padrão ponta-onda contínua no sono reforça o diagnóstico de epilepsia rolândica atípica, hoje bastante discutida na literatura, se seria uma síndrome independente ou parte de um continuum relacionado ao espectro das epilepsias com descargas centro-temporais $(6,11,13)$.

A intensidade das descargas epileptiformes durante o sono, ocupando pelo menos $50 \%$ do traçado remete à possibilidade do diagnóstico de uma das síndromes epilépticas com padrão de POCS (14). As epilepsias com padrão POCS geralmente apresentam um curso clínico mais grave, com deterioração cognitiva, configurando uma encefalopatia epiléptica (15). Atualmente acredita-se que nos pacientes que apresentem o padrão POCS, a presença de uma atividade epiléptica contínua e prolongada possa desencadear alterações neuropsicológicas e comportamentais, possivelmente por causar um dano à plasticidade cortical, com prejuízo no desenvolvimento cognitivo (16). No caso relatado por nós não houve uma encefalopatia epiléptica, por não haver comprometimento cognitivo ou comportamental, apesar da existência do padrão POCS durante boa parte da evolução. Não é possível prever, caso a Oxcarbazepina ou Lamotrigina, drogas que agem nos canais de sódio, fossem utilizadas por tempo mais prolongado, se haveria uma persistência do padrão POCS ou se haveria um comprometimento cognitivo. Caraballo et al (2013) observaram em estudo multicêntrico não haver diferenças eletro-clínicas entre os pacientes com índices de espícula-onda acima de $85 \%$ e aqueles com índices abaixo desse valor do traçado em sono(17), o que pode significar que esta expressão fenotípica não seja o único determinante para uma evolução pior, mas que outros fatores gené- 
ticos possam também ter um papel determinante(10). Mais importante que uma definição diagnóstica do tipo de epilepsia é a identificação de fatores que possam contribuir para uma evolução desfavorável. Saltik et al (2005) estudaram pacientes com epilepsia parcial idiopática para tentar identificar características clínicas e eletroencefalográficas capazes de prever uma evolução desfavorável para uma encefalopatia epiléptica com padrão de POCS. As principais características clínicas identificadas foram o aumento na frequência e na variabilidade das crises epilépticas, o surgimento de novos tipos de crise e alterações cognitivas e/ou comportamentais. No EEG, a presença de um alentecimento contínuo na mesma região do principal foco epileptogênico, além de propagação das descargas epileptiformes para áreas adjacentes, especialmente no hemisfério oposto. (18). O caso que relatamos apresentava todas essas características clínicas e eletroencefalográficas, com exceção das alterações cognitivas e comportamentais. Fejerman et al (2000), através da análise dos traçados eletroencefalográficos e tipos de crises de pacientes com epilepsia rolândica, não conseguiram identificar alterações que pudessem predizer uma evolução atípica (7).

A resposta atípica com o uso de drogas de primeira escolha para as epilepsias focais é outro ponto a ser considerado para uma evolução desfavorável. A Oxcarbazepina, assim como a Carbamazepina e a Lamotrigina, atuam através do bloqueio dos canais de sódio voltagem-dependente. São relatados casos de deterioração cognitiva e comportamental associadas ao agravamento da epilepsia e padrão POCS em pacientes com epilepsia rolândica, após a administração de Carbamazepina, com melhora evidente após a descontinuação da droga (8), porém o risco de agravamento é muito pequeno, não podendo descartar a possibilidade de ser uma coincidência com os períodos de piora espontânea no curso da epilepsia. (9). Fujii et al (2010) observaram que nos casos de epilepsia parcial atípica benigna, a Carbamazepina pode eventualmente agravar as crises, principalmente crises mioclonicas negativas, mas não deve ser considerado o agente causal na evolução atípica da epilepsia rolândica(6). Outros autores também relatam exacerbações de crises e surgimento de manifestações atípicas, incluindo POCS, em pacientes com epilepsia rolândica tratados com Oxcarbazepina $(19,20)$.

Nossa paciente apresentou uma piora progressiva na frequência de crises, com surgimento de um novo tipo de crise (as mioclonias negativas) e evolução do padrão eletroencefalográfico para ponta-onda contínua no sono durante o tratamento com Oxcarbazepina e Lamotrigina. A melhora transitória, após a troca da medicação para Lamotrigina, pode ter ocorrido por ser uma droga de amplo espectro, com outros mecanismos de ação, além da ação de bloqueio dos canais de sódio. Após a substituição destas drogas pelo Suthiame, cujo principal mecanismo de ação é a inibição da anidrase carbônica, houve uma resposta eletro-clínica dissociada. A paciente apresentou rapidamente controle das crises, porém a melhora eletroencefalográfica foi mais lenta e intermitente, com recidiva do padrão POCS, sem correspondência clínica. A ausência de comprometimento neuropsiquiátrico, o controle das crises e posterior redução progressiva das descargas epileptiformes sugerem que existe uma expressão fenotípica independente, determinado por um único gene ou por vários genes.

\section{CONCLUSÃO}

A epilepsia benigna da infância com descargas centro-temporais possivelmente faz parte de um espectro clínico, com variação nas manifestações clínicas e eletroencefalográficas. É necessário estar atento para o reconhecimento de manifestações clínicas e eletroencefalográficas atípicas e identificar fatores que possam contribuir para uma evolução desfavorável, inclusive precipitadas pelas drogas antiepilépticas.

A escolha da medicação deve ser reavaliada quando a evolução for desfavorável, levando-se em conta a possibilidade de haver uma contribuição da medicação na piora clínica.

O tratamento deve objetivar o controle das crises e a preservação das funções cognitivas. Uma abordagem terapêutica agressiva na tentativa de melhora do padrão eletroencefalográfico só estaria justificada quando há prejuízo neuropsicológico, principalmente se houver correlação entre as alterações eletroencefalográficas e a deterioração cognitiva.

\section{REFERÊNCIAS}

1. ILAE. Proposal for revised classification of epilepsies and epileptic syndromes. Commission on Classification and Terminology of the International League Against Epilepsy. Epilepsia, Malden, v. 30, p. 389399, Aug 1989.

2. Panayiotopoulos, C. P. et al. Benign childhood focal epilepsies: assessment of established and newly recognized syndromes. Brain, Oxford, v. 131, p.2264-2286, Sep 2008.

3. Marie-Noëlle, M.; Filippini, M. Neuropsychological Findings in Rolandic Epilepsy and Landau-Kleffner Syndrome. Epilepsia, Malden, v. 47, Suppl. 2, p. 71-75, Nov 2006.

4. Gobbi, G.; Boni, A.; Filippini, M. The Spectrum of Idiopathic Rolandic Epilepsy Syndromes and Idiopathic Occipital Epilepsies: From the Benign to the Disabling. Epilepsia, Malden, V.47, Suppl.2, p. 62-66, Nov 2006

5. Aicardi, J.; Chevrie, J.J. Atypical benign partial epilepsy of childhood. Dev Med Child Neurol, London, v.24, p. 281-292, Aug 1982.

6. Fujii, A. et al. Atypical Benign Partial Epilepsy: Recognition Can Prevent Pseudocatastrophe. Pediatr Neurol, New York, v.43, p. 411-419, Dec 2010.

7. Fejerman, N.; Caraballo, R.; Tenembaum, S. N. Atypical Evolutions of Benign Localization-Related Epilapsies in Children: Are They Predicta- 
ble?. Epilepsia, Malden, v.41, n.4, p. 380-390, April 2000

8. Prats, J. M. et al. Antiepileptic Drugs and Atypical Evolution of Idiopathic Partial Epilepsy. Pediatr Neurol, New York, v.18, p. 402-406, May 1998.

9. Corda, D. et al. Incidence of Drug-Induced Aggravation in Benign Epilepsy with Centrotemporal Spikes. Epilepsia, Malden, v.42, n.6, p. 754759, June 2001

10. Lemke, J. R. et al. Mutations in GRIN2A cause idiopathic focal epilepsy with rolandic spikes. Nature Genetics, New York, v.45, n.9, p. 1067-1072, Sep 2013.

11. Fejerman, N. Atypical rolandic epilepsy. Epilepsia, Malden, v.50, suppl 7, p. 9-12, Aug 2009.
12. Verrotti, A. et al. Typical and atypical Rolandic epilepsy in childhood: a follow up study. Pediatr Neurol, New York, v.26,p.26-29, Jan 2002

13. Hahn, A. et al. Atypical "Benign" Partial Epilepsy or Pseudo-Lennox Syndrome. Part I: Symptomatology and Long-Term Prognosis. Neuropediatrics, Stuttgart, v.32, p. 1-8, Feb 2001.

14. Scheltens-de Boer, M. Guidelines for EEG encephalopathy related to ESES/CSWS in children. Epilepsia, Malden, v. 50 (Suppl 7), p. 13-17, Aug 2009.

15. Berg, A. T. et al. Revised terminology and concepts for organization of seizures and epilepsies: Report of the ILAE Commission on Classification and Terminology, 2005-2009. Epilepsia, Malden, v.51, n.4, p. 676-685, April 2010. 
Padrão de ponta-onda Contínua durante o sono na epilepsia parcial idiopática:

relato de um caso sem alterações neuropsiquiátricas

16. Tassinari, C. A. et al. Encephalopathy with status epilepticus during slow sleep: "The Penelope syndrome". Epilepsia, Malden, v.50, (Suppl 7), p. 4-8, Aug 2009.

17. Caraballo, R. H. et al. Encephalopathy with status epilepticus during sleep or continuous spikes and waves during slow sleep syndrome: A multicenter, long-term follow-up study of 117 patients. Epilepsy Research, Amsterdam, v. 105, p. 164-173, July 2013.
18. Saltik, S. et al. A Clinical and EEG Study on Idiopathic Partial Epilepsies with Evolution into ESES Spectrum Disorders. Epilepsia, Malden, v. 46, n.4, p. 524-533, April 2005.

19. Grosso, S. et al. Oxcarbazepine and atypical evolution of benign idiopathic focal epilepsy of childhood. European Journal of Neurology, Oxford, v.13, n.10, p. 1142-1145, Oct 2006.

20. Chapman, K.; Holland, K.; Erenberg, G. Seizure exacerbation associated with oxcarbazepine in idiopathic focal epilepsy of childhood. Neurology, Minneapolis, v.61, p. 1012-1013, Oct 2003.

Submetido em 13.11.2013

Aceito em 20.12.2013. 